\title{
I feel what you feel: empathy and placebo mechanisms for autonomous virtual humans
}

\author{
Julien Saunier, Hazaël Jones, Domitile Lourdeaux \\ Laboratoire Heudiasyc \\ UMR CNRS 6599 \\ Université de Technologie de Compiègne \\ $\{$ jsaunier, joneshaz, dlourdea\}@hds.utc.fr
}

\begin{abstract}
Computational modeling of emotion, physiology and personality is a major challenge in order to design believable virtual humans. These factors have an impact on both the individual behavior and the collective one. This requires to take into account the empathy phenomenon. Furthermore, in a crisis simulation context where the virtual humans can be contaminated by radiological or chemical substances, empathy may lead to placebo or nocebo effects.

Stemming from works in the multiagent systems domain, our virtual human decision process is designed as an autonomous agent. It has been shown that the environment can encapsulate the responsibility of spreading a part of the agent state. The agent has two parts, its mind and its body. The mind contains the decision process, and is the autonomous part of the agent. The body is influenced by the mind, but controlled by the environment. One may try to fly, it does not mean one can.
\end{abstract}

\section{Introduction}

The context of this research is a project of crisis simulation for the improvement of multi-institutional response to terrorist attacks. The goal is to propose a training tool for both security and rescue teams (command and action roles). Civilians are virtual autonomous humans / agents represented in a virtual environment.

In order to improve crisis simulation, designers have to take into account personality, emotion and physiology in decision making $[1,20]$. However, these mechanisms are described through many conflicting and/or shallow-defined models [21]. Another difficulty is that scripted scenarios limit the exercise replayability, but non-scripted simulation is hard to achieve and resource hungry. Furthermore, being a training tool, the sequence of events has to be explainable.

Multiagent systems (MAS) are a way to simulate virtual humans via cognitive agents. There are two main approaches to obtain an intelligent behavior : imitate the cognitive process [6], or manipulate a set of observed behaviors [12]. Our research follows an hybrid path that simulates interdependencies in behavior choice, in order to both explain what cognitive factors lead to simulated situations and keep the agents complexity coherent with the simulation needs. This architecture uses ergonomics task models, extending [5]. 
In this article, we focus on the social part of the agents. Collective behavior is not an aggregation of individual behaviors [11]; in particular because of empathy [16] and, combined with biased reasoning, placebo/nocebo effects. Empathy is "the intellectual identification with or vicarious experiencing of the feelings, thoughts, or attitudes of another" (Dictionary). Basically, it means that the agents influence each other through some kind of affective interaction. In the crisis context where the virtual humans can be contaminated by radiological or chemical substances, empathy may lead to nocebo effects [2]: someone believing he is contaminated and possibly even showing physical symptoms although he is safe.

In section 2, we show the motivations of our architecture, both at the agent and multi-agent level. Section 3 is an overview of our architecture named PEP $\rightarrow$ BDI. Section 4 describes the inter-agent empathy mechanism and how it can lead to nocebo. We show the results of several experiments in section 5, and draw conclusions and perspectives in section 6 .

\section{State of the art}

\subsection{Architectures}

Most of the architectures integrating emotions and personalities focus on facial modelling and/or user-virtual human interaction. Conversely, in our research we are interested in high-level decision process modelling, while biomecanics are managed through ad hoc extern modules.

In cognitive modeling, BDI architecture [19,7] is often used for its intuitive representation of agent reasoning. The reasoning is decomposed in modules for a clear structure. For these reasons, we decided to base our model on this architecture. However, in the original model, emotions, personality and physiology are not taken in account in the decision process. From this observation, Jiang and al. developped the eBDI architecture [9] that introduce emotion in a BDI architecture. However, this approach does not consider personality and physiology aspects.

In emotions modeling, several works have been proposed: Gratch [6] proposes the most accomplished current model for agent emotions representation. However, its formalism is complex and fully dedicated to emotions representation. As a consequence, this model is not easily adaptable and needs a complex calibration. It is adapted to domains where a subtle individual emotion representation is needed (facial expression representation, dialog management, ...) for a limited number of agent. In our project, we want to model individual and emergent collective emotions for many agents.

These objectives are the same as Silverman et al. [20]. They propose a complete architecture that considers agent emotions, physiology, personality and culture. This architecture is fully integrated and the functional separation of modules is static, in order to experiment unitary tests. This approach is complementary of ours. On one hand, we evaluate emotion, personality and physiology in a well-known architecture (BDI). On the other hand, we also explore how the environment can be exploited to provide empathy mechanisms. 
DETT ${ }^{1}$ agent architecture [17] deals with the link between personality and emotions in a straightforward way. It is based on properties defined in OCC model [15]. Especially, DETT defines tendencies, that is the inclination of an agent to feel and to update its emotions in time. However, there are two limits to this approach: DETT is not explanatory (there is a direct link between emotion and action, but no high-level decision), and it models only two emotions (fear and bravery) in relation with two personality aspects (cowardice and temper).

\subsection{Social phenomena}

Studies from the psychology field, such as [3], highlight the individual and social dimensions of crisis. At the individual level, disasters engender high levels of stress, which can etither be adapted or overwhelming. Adapted stress mobilizes the mental and physiological capacities, while overwhelming stress exhausts the energetic reserves through one of its four modalities: stuporous inhibition, uncontrolled agitation, individual panic flight, and automatic behavior. Symmetrically, at the collective level, bevahiors can be adjusted or maladjusted. Maladjusted behaviors such as panic and violence may arise when the rescue teams are feebly organized and when the victims believe they are poorly informed or treated.

Mutual awareness [4] and empathy are necessary for collective behavior to appear [11]. Empathy is the low-level mechanism which enables the agents to perceive each other physical and emotional state. At a higher level, mutual awareness involves a symbolic representation of the others activities.

Stemming from works in the multiagent systems domain, our virtual human decision process is designed as an autonomous agent. The environment has been recently put forward as a first-order abstraction [22] which can encapsulate the responsibility of spreading a part of the agent state.

Following this principle, the agent has two parts: its mind and its body [18]. The mind contains the decision process, and is the autonomous part of the agent. The body is influenced by the mind, but controlled by the environment. One may try to fly, it does not mean one can. Practically, the agent state is public, and its modification is regulated by the environment.

This model is useful in term of representation (reactive body, incertitude of an agent on its contamination state, ...). In terms of computation, all the calculation normally done by the agent is delegated to the environment.

\section{System architecture}

\subsection{Global overview}

Figure $1^{2}$ shows our global architecture. The virtual environment is an extern module called EVE which manages the 3D representation of the scene. The SMA platform manages the agents life cycle and communications, and each virtual human is driven by an agent.

\footnotetext{
${ }^{1}$ Disposition, Emotion, Trigger, Tendency

${ }^{2}$ Screenshot: EVE(C), EMI Informatique.
} 


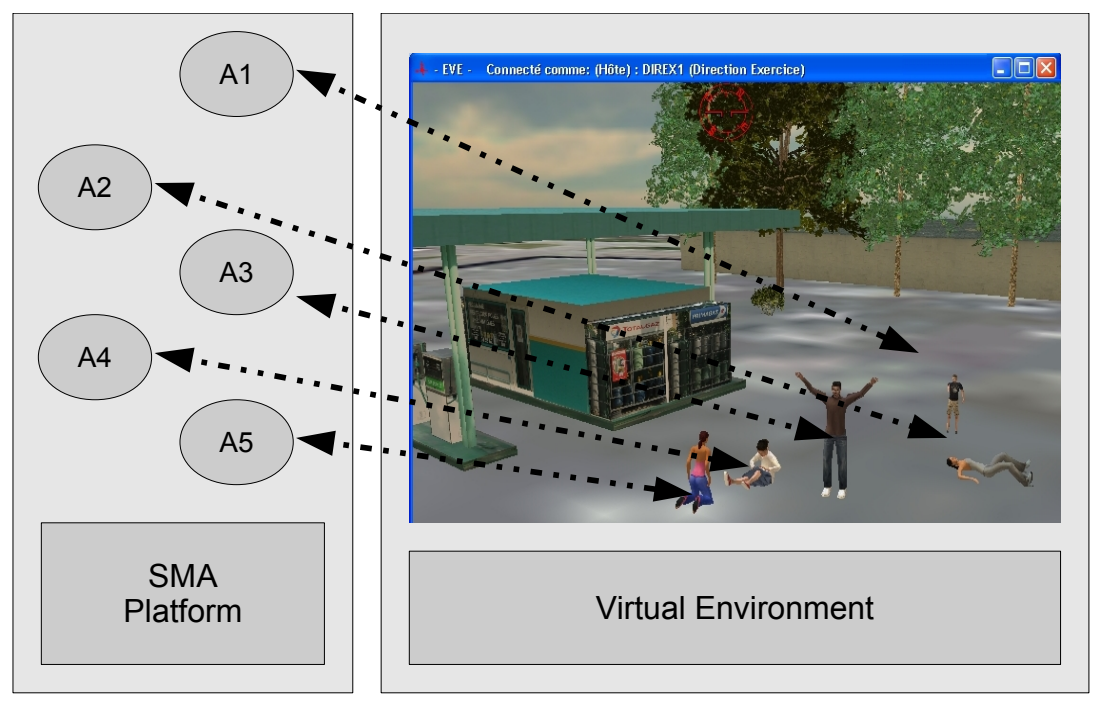

Fig. 1. Global Architecture.

The virtual environment and the SMA platform are synchronized in order to ensure consistency during the simulation.

\subsection{Agent architecture}

We introduce briefly the architecture PEP $\rightarrow$ BDI which is an extension of eBDI framework [9]. The goal of this model is to consider emotion, personality and physiology of an agent in its decision process.

Figure 2 shows our agent architecture.

The agent takes new information (perception, message and body) from the environment. This new information generates immediate emotions, and the agent changes its beliefs in function of its emotions. Physiological informations are updated in the same way as beliefs. We show in section 4 how it is used to model placebo effects.

The selection of desire and intentions is similar to the classical BDI scheme except for the emotion and physiology influence. Once intentions are selected, the agent updates its emotions again. If its emotions change, it updates again its beliefs, physiology, desires and intentions. Finally, it plans its actions and executes its new plan.

Basically, emotions are based on OCC [15]. Emotions are grouped by pair like for example pride and shame. We take into account emotions relevant to our crisis simulation: fear and hope, anger and gratefulness, shame and pride, reproach and trust. In this article, the focus is put on empathy in a terrorist attack simulation. Hence, relevant factors are fear, anger and stress (physiological parameter).

Personality is formed by parameters that indicate personality traits [13]. In a crisis situation, agents evolve on a short time period (a few hours) where only some prominent 


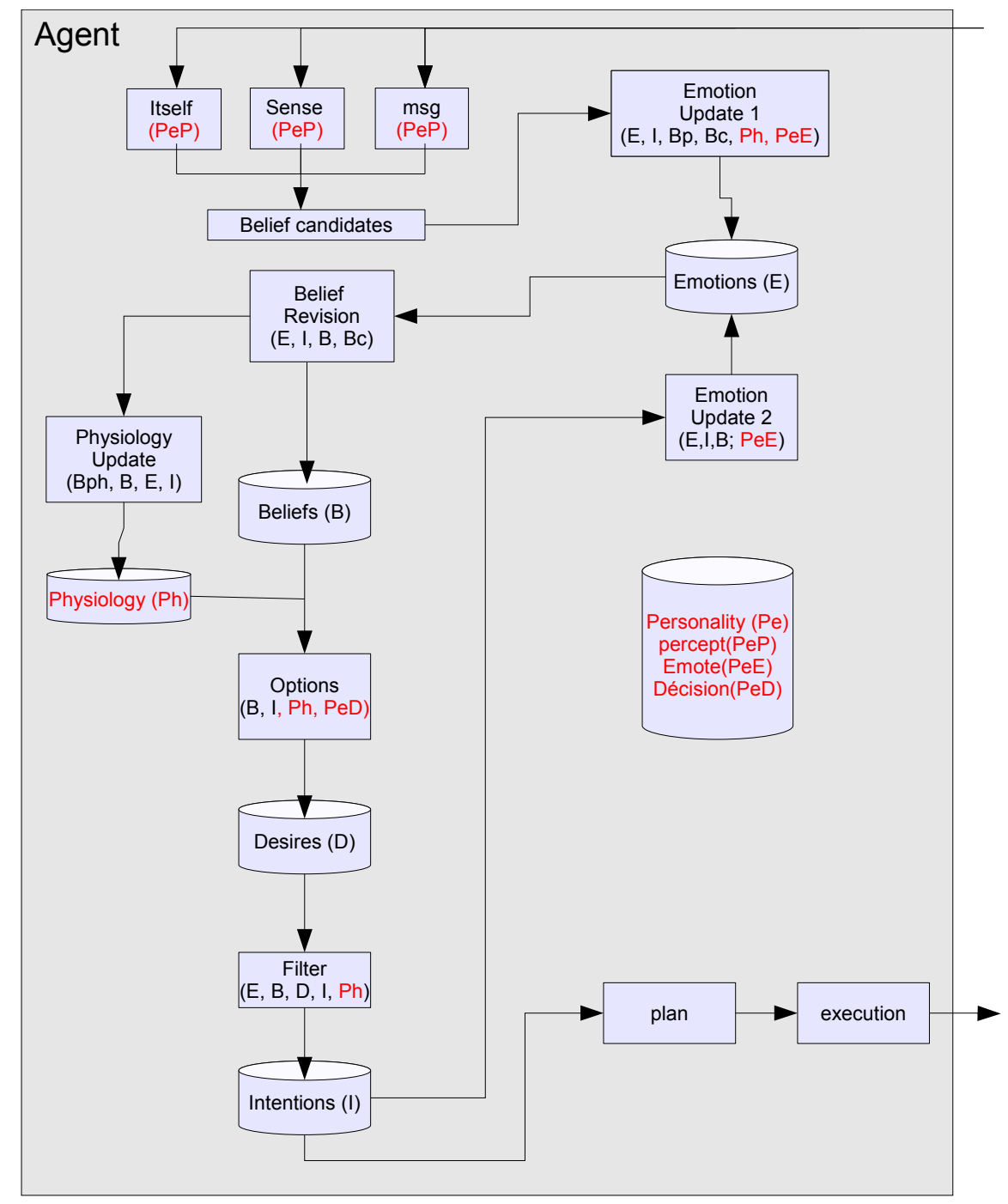

Fig. 2. Agent Architecture

behavior elements are expressed. As a consequence, we chose to simplify the personality model and to use only personality traits relevant for the simulation. The personality of an agent does not evolve during the simulation. 


\section{State spreading and its influence on the agents}

\subsection{Empathy}

As stated in the introduction, empathy enables the agents to be impacted by the other agents state. Proximity, either spatial or psychological, is a requirement for empathy to take place. For example, in Fig. 3, $a_{1}$ and $a_{2}$ are very close, while $a_{3}$ is further away. While $a_{2}$ becomes angry, sharing $a_{1}$ 's mood, the state of $a_{3}$ is not modified.

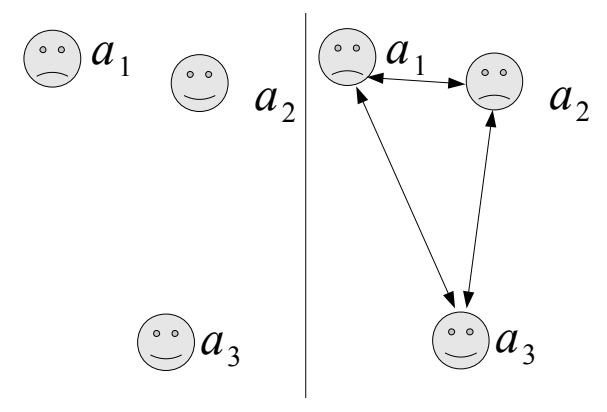

Fig. 3. Left: initial states. Right: new states.

The functional separation of mind and body means that the agent knows its physical / emotional state, but can only influence it. In fact, its emotions will be updated through two mechanisms:

- Internal dynamics: emotions and physiology evolve in function of time towards an equilibrium.

- External dynamics: emotions and physiology vary in function of events and stimuli.

Internal dynamics are managed by the agent itself. It is calculated as: emotion $=$ emotion $\times$ emotion_tendency

External dynamics are managed by the perception function of the environment, in order to give the right information to the right agent(s). Concerning the empathy mechanisms specifically, the environment updates regularly the agents state (figure 4).

The empathy manager gets the current state of the agent. It updates accordingly its state of the world. The state of the world contains the body properties of each agent. Then, it calculates the effects of empathy on the agents neighbours in function of their previous state and their tendency to empathy. Finally, the environment spreads these into the concerned agents bodies.

The calculus is:

stress $=\frac{1}{\text { dist(origin,target) }}$ stress $* t_{e}$ with $\operatorname{dist}(a, b)$ the distance between $a$ and $b, t_{e}$ (target) the empathy tendency of the target.

We calculate in the same way the two emotions, namely fear and anger. 


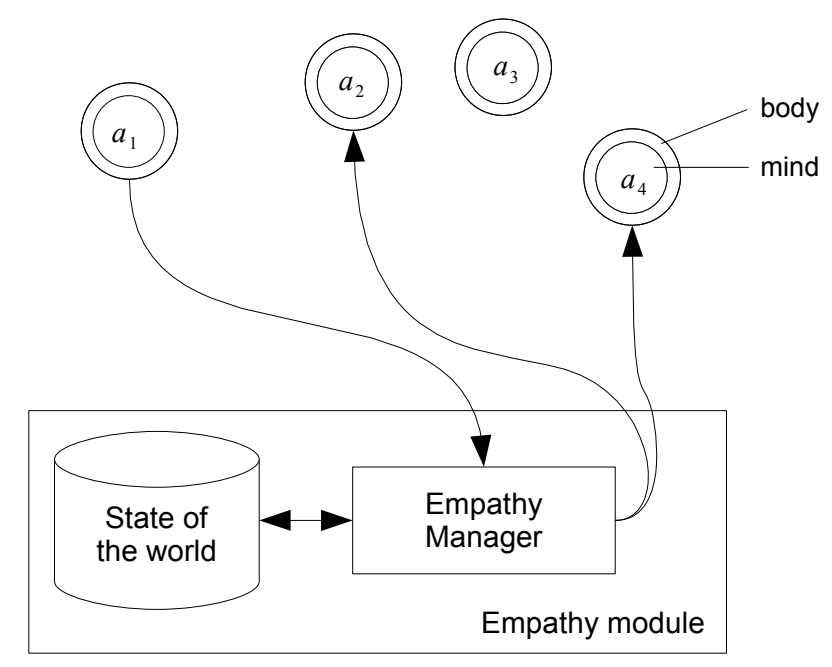

Fig. 4. Environment empathy module and agents interactions

\subsection{Placebo}

In our crisis scenario, part of the civilians inhale and/or touch chemical and radiological substances. These civilians develop symptoms quickly, which range from itching to suffocation. A major problematic for the rescuers is to isolate the contaminated victims. However, a part of the population will mimic reactions to the toxics without real exposure $[14,10]$.

Three categories of factors are involved in the nocebo effect: personality, physiology, emotion and beliefs.

Personality- Studies show that in clinical experiments 30 to $40 \%$ of the patients are placebo-responsive, but that there is no obvious personality trait which may lead to placebo or nocebo responsiveness [8].

Two main personality factors were identified:

- Optimism: one who believes in a positive outcome tends to be less responsive than one who does not.

- Empathy: one who is prone to assimilate the others feelings can also share perceptible symptoms.

Physiology- Although optimism and empathy tendency play a role in placebo responsiveness, of greater importance are immediate situational and interpersonal factors.

The first of these factors is stress. Nervous tension is a consequence of general adaptation. It is influenced [20] by temporal pressure, tiredness, positive or negative events and actions success / failure. Generally, it will follow the same curve as emotions. 
Beliefs- New information can be obtained by perception (sight, hearing, smelling, ...), by communication (messages) or by sensing the semi-controlled body (injury, tiredness, ... ). Primary emotions are a direct reaction to a percept. For example, if an agent perceives several agents suffocating, it will feel fear.

In our representation, emotions $E$ and personality $P e$ have an impact on the way the new beliefs are interpreted. This leads the agent to either modify the input, for example a coward agent is more inclined to believe the situations to be dangerous, or build biased beliefs.

In our simulation, because of the crisis situation, it is important for agents to have a risk representation. This includes the agents beliefs about the other agents contamination, assessed through visible symptoms, and about itself. Furthermore, emotions influence the agents survival prediction belief.

The agents starts showing symptoms when it has persuaded itself it is contaminated.

Calculation- All these factors increase or decrease the probability for the agent to be placebo-responsive, but there is no predicitive rule. The calculation is realized when an event likely to cause placebo happens. These events are:

- Information or rumor about the presence of a contaminating substance

- Addition of a new belief evaluating an agent to be contaminated

- Survival prediction belief crossing the lower threshold

Stress and emotions are evaluated in $[0,100]$, emotion tendancies belong to $[1-$ $t, 1+t]$. The calculation itself is realized as follows:

contam $($ itself $)=\left(\sum \frac{1}{\operatorname{dist}(\text { itself,agent })} \operatorname{contam}(\right.$ agent $)+$ stress + optimism $*$ survival $_{p}$ rediction $) \times$ $t_{e} \times p$ with $p$ a probability.

If the agents believes it is contaminated, it mimics the symptoms of nearby agents.

\section{Experiments}

We have run experiments using the multiagent platform MadKit ${ }^{3}$.

\section{1 empathy}

White are low-stressed, then yellow - orange - red. Revoir couleurs pour lisibilité.

Random initialization, we can see in the final state that clusters of agents with the same stress level are forming, because the agents are sensible to the state of their neighbors. Furthermore, the clusters do not rely exclusively on the agents stressability.

Faire tests évolution sans empathy/avec.

\footnotetext{
${ }^{3}$ www.madkit.org
} 


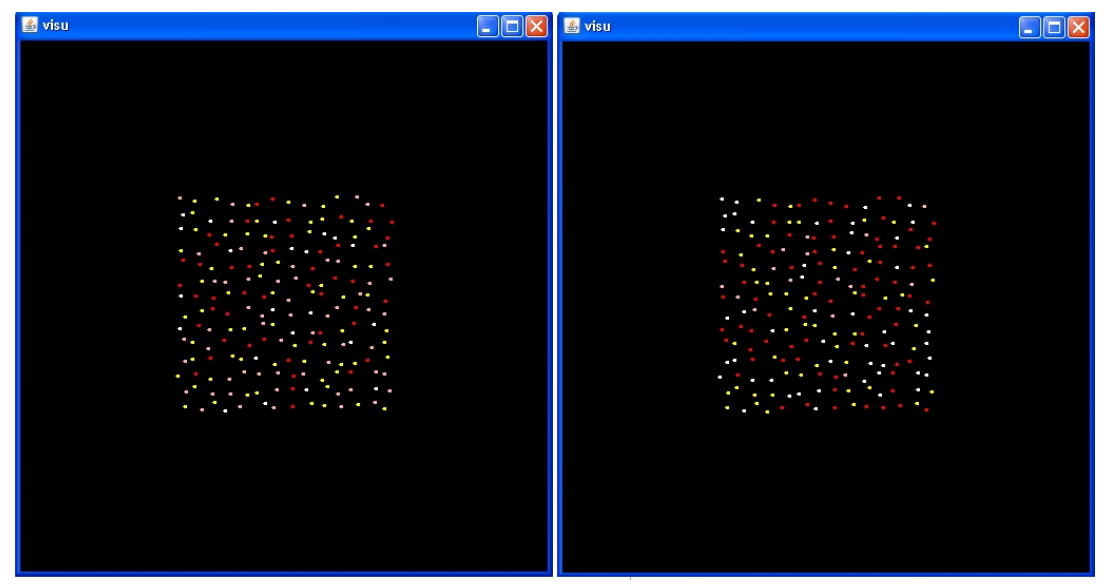

Fig. 5. Left: initial state. Right: final state

\section{2 contamination}

\section{Conclusion and perspectives}

Simulation of human behavior, in particular in a crisis situation, needs to consider physiology, personality and emotion in order to obtain plausible behavior. Furthermore, collective behavior simulation requires the instantiation of social phenomena such as empathy and nocebo effects. An originality of this work is to use the environment to spread the states of the agents. This mechanism enables the representation of complex social behavior.

Further work include a thorough validation of simulated behavior at global level and individual levels. This work of calibration will need psychology study and users experiments to improve our agents behaviors.

\section{References}

1. J. Bates. The role of emotion in believable agents. Commun. ACM, 37(7):122-125, 1994.

2. H. Benson. The nocebo effect: History and physiology,. Preventive Medicine, 26(5):612 $615,1997$.

3. L. CROCQ. Special teams for medical/psychological intervention in disaster victims. World Psychiatry, 1(3):154, 2002.

4. J. Dugdale, J. Pavard, and B. Soubie. A pragmatic development of a computer simulation of an emergency call center. In Designing Cooperative Systems: The Use of Theories and Models, pages 241-256. IOS Press, 2000.

5. L. Edward, D. Lourdeaux, D. Lenne, J. Barthes, and J. Burkhardt. Modelling autonomous virtual agent behaviours in a virtual environment for risk. IJVR : International Journal of Virtual Reality, 7(3):13-22, September 2008.

6. J. Gratch and S. Marsella. A domain-independent framework for modeling emotion. Cognitive Systems Research, 5(4):269 - 306, 2004. 
7. A. Haddadi and K. Sundermeyer. Belief-desire-intention agent architectures. pages 169-185, 1996.

8. A. Harrington. The placebo effect: an interdisciplinary exploration. Harvard University Press, 1997.

9. H. Jiang, J. M. Vidal, and M. N. Huhns. Ebdi: an architecture for emotional agents. In AAMAS '07: Proceedings of the 6th international joint conference on Autonomous agents and multiagent systems, pages 1-3, New York, NY, USA, 2007. ACM.

10. T. Lacy and D. Benedek. Terrorism and Weapons of Mass Destruction: Managing the Behavioral Reaction in Primary Care. Southern Medical Journal, 96(4):394, 2003.

11. G. Le Bon. The Crowd: A Study of the Popular Mind. 1895.

12. P. Maes. The agent network architecture (ana). SIGART Bull., 2(4):115-120, 1991.

13. R. McCrae and O. John. An introduction to the five-factor model and its applications. Journal of Personality, 60:175-215, 1992.

14. S. Noy. Minimizing casualties in biological and chemical threats (war and terrorism): the importance of information to the public in a prevention program. Prehospital and Disaster Medicine, 19(1):29-36, 2004.

15. A. Ortony, G. L. Clore, and A. Collins. The Cognitive Structure of Emotions. Cambridge University Press, July 1988.

16. A. Paiva, J. Dias, D. Sobral, R. Aylett, P. Sobreperez, S. Woods, C. Zoll, and L. Hall. Caring for agents and agents that care: Building empathic relations with synthetic agents. In AAMAS '04: Proceedings of the Third International Joint Conference on Autonomous Agents and Multiagent Systems, pages 194-201, Washington, DC, USA, 2004. IEEE Computer Society.

17. H. V. D. Parunak, R. Bisson, S. Brueckner, R. Matthews, and J. Sauter. A model of emotions for situated agents. In AAMAS '06: Proceedings of the fifth international joint conference on Autonomous agents and multiagent systems, pages 993-995, New York, NY, USA, 2006. ACM.

18. E. Platon, N. Sabouret, and S. Honiden. Tag interactions in multiagent systems: Environment support. In Proceedings of Environment for Multi-Agent Systems, Workshop held at the Fifth Joint Conference in Autonomous Agents and Multi-Agent Systems, volume 4389 of Lecture Notes in Artificial Intelligence, pages 106-123. Springer Verlag, 2007.

19. A. S. Rao and M. P. Georgeff. Modeling rational agents within a BDI-architecture. In J. Allen, R. Fikes, and E. Sandewall, editors, Proceedings of the 2nd International Conference on Principles of Knowledge Representation and Reasoning (KR'91), pages 473-484. Morgan Kaufmann publishers Inc.: San Mateo, CA, USA, Apr. 1991.

20. B. G. Silverman, M. Johns, J. Cornwell, and K. O'Brien. Human behavior models for agents in simulators and games: Part i: Enabling science with pmfserv. Presence, 15(2):139 - 162, 2006.

21. A. Sloman. Beyond shallow models of emotion. In Cognitive Processing: International Quarterly of Cognitive Science, pages 177-198, 2001.

22. D. Weyns, A. Omicini, and J. Odell. Environment as a first-class abstraction in multi-agent systems. Autonomous Agents and Multi-Agent Systems, 14(1):5-30, Feb. 2007. Special Issue on Environments for Multi-agent Systems. 